Article

\title{
Correlations between Maternal, Breast Milk, and Infant Vitamin B12 Concentrations among Mother-Infant Dyads in Vancouver, Canada and Prey Veng, Cambodia: An Exploratory Analysis
}

\author{
Philip Chebaya ${ }^{1,2}$, Crystal D. Karakochuk ${ }^{1,2}$, Kaitlin M. March ${ }^{1,2}$, Nancy N. Chen ${ }^{1,2}$, \\ Rosemary A. Stamm ${ }^{3}$, Hou Kroeun ${ }^{4}$, Prak Sophonneary ${ }^{5}$, Mam Borath ${ }^{6}$, \\ Setareh Shahab-Ferdows ${ }^{7}$, Daniela Hampel ${ }^{7,8}$, Susan I. Barr ${ }^{1}$, Yvonne Lamers ${ }^{1,2}$, \\ Lisa A. Houghton ${ }^{3}$, Lindsay H. Allen ${ }^{7,8}$, Tim J. Green 1,2,9,* and Kyly C. Whitfield 1,2,10 \\ 1 Food, Nutrition, and Health, The University of British Columbia, Vancouver, BC V6T 1Z4, Canada; \\ philipchebaya@gmail.com (P.C.); crystal.karakochuk@alumni.ubc.ca (C.D.K.); \\ marchkmr@gmail.com (K.M.M.); nnchen30@gmail.com (N.N.C.); susan.barr@ubc.ca (S.I.B.); \\ yvonne.lamers@ubc.ca (Y.L.); kyly.whitfield@msvu.ca (K.C.W.) \\ 2 BC Children's Hospital Research Institute, Vancouver, BC V6H 3N1, Canada \\ 3 Department of Human Nutrition, University of Otago, Dunedin 9016, New Zealand; \\ rosemary.stamm@otago.ac.nz (R.A.S.); lisa.houghton@otago.ac.nz (L.A.H.) \\ 4 Helen Keller International—Cambodia Country Office, Phnom Penh 12301, Cambodia; hkroeun@hki.org \\ 5 National Nutrition Programme, Maternal and Child Health Centre, Ministry of Health, Phnom Penh 12202, \\ Cambodia; sophonprak@gmail.com \\ 6 National Sub-Committee for Food Fortification, Ministry of Planning, Phnom Penh 12000, Cambodia; \\ borathmam@yahoo.com \\ 7 US Department of Agriculture, ARS Western Human Nutrition Research Centre, University of California, \\ Davis, CA 95616, USA; setti.shahab-ferdows@ars.usda.gov (S.S.-F.); daniela.hampel@ars.usda.gov (D.H.); \\ lindsay.allen@ars.usda.gov (L.H.A.) \\ 8 Department of Nutrition, University of California, Davis, CA 95616, USA \\ 9 South Australia Health and Medical Research Institute, Adelaide, South Australia 5000, Australia \\ 10 Department of Applied Human Nutrition, Mount Saint Vincent University, Halifax, NS B3M 2J6, Canada \\ * Correspondence: tim.green@sahmri.com; Tel.: +61-8-8128-4000
}

Received: 29 December 2016; Accepted: 9 March 2017; Published: 12 March 2017

\begin{abstract}
Vitamin B12 plays an essential role in fetal and infant development. In regions where animal source food consumption is low and perinatal supplementation is uncommon, infants are at risk of vitamin B12 deficiency. In this secondary analysis, we measured total vitamin B12 concentrations in maternal and infant serum/plasma and breast milk among two samples of mother-infant dyads in Canada (assessed at 8 weeks post-partum) and in Cambodia (assessed between 3-27 weeks post-partum). Canadian mothers $(n=124)$ consumed a daily vitamin B12-containing multiple micronutrient supplement throughout pregnancy and lactation; Cambodian mothers $(n=69)$ were unsupplemented. The maternal, milk, and infant total vitamin B12 concentrations (as geometric means (95\% CI) in pmol/L) were as follows: in Canada, 698 (648,747), 452 (400, 504), and 506 (459, 552); in Cambodia, 620 (552, 687), $317(256,378)$, and $357(312,402)$. The majority of participants were vitamin B12 sufficient (serum/plasma total B12 > $221 \mathrm{pmol} / \mathrm{L}$ ): $99 \%$ and $97 \%$ of mothers and $94 \%$ and $84 \%$ of infants in Canada and Cambodia, respectively. Among the Canadians, maternal, milk, and infant vitamin B12 were all correlated $(p<0.05)$; only maternal and infant vitamin B12 were correlated among the Cambodians $(p<0.001)$.
\end{abstract}

Keywords: vitamin B12 (cobalamin); lactation; human milk; Canada; Cambodia 


\section{Introduction}

Vitamin B12 is found only in animal-source or fortified foods [1], so deficiency is most common in those who consume vegan diets [2] or in populations in low- and middle-income countries where animal foods are not available or affordable [3]. Maternal vitamin B12 status in pregnancy strongly influences infant vitamin B12 status [4]; breast milk vitamin B12 content is reduced when maternal vitamin B12 status is poor [5]. Since exclusive breastfeeding is recommended for the first 6 months of life [6], infants rely solely on their stores and breast milk for vitamin B12 during this time [2]. Attaining adequate vitamin B12 status in infancy-a time of rapid growth and development-is essential for normal cognitive development in infants [7]. Deficiency, which typically presents in infants between 4 and 10 months but may appear within months of birth, can lead to failure to thrive, developmental regression [2], and severe neuropathy [8].

There is currently little published data describing blood and milk vitamin B12 concentrations of purportedly well-nourished mother-infant dyads [4]. For instance, there is currently no agreed-upon cut-off for vitamin B12 deficiency in breast milk; although a cut-off of $<362 \mathrm{pmol} / \mathrm{L}$ was used to define deficiency previously, it was developed over 25 years ago using older laboratory methods that may have incorrectly measured vitamin B12 analogues, and included only 19 milk samples [9,10]. Greibe et al. measured plasma vitamin B12 concentrations among 60 Danish mother-infant dyads, and breast milk vitamin B12 among a subset of 25 mothers at 2 weeks, 4 months, and 9 months postpartum [11]. The majority of those women were consuming daily vitamin B12-containing multivitamin supplements. Moderate correlations ( $r=0.27$ to 0.52$)$ were found between maternal and infant vitamin B12 concentrations at all three time-points. Milk vitamin B12 concentrations, however, fluctuated considerably throughout lactation, and were only significantly correlated with maternal and infant plasma vitamin B12 concentrations at 4 months postpartum [11]. In contrast, the authors of an earlier study reported that breast milk B12 concentrations remained relatively constant after falling from their highest levels in colostrum [12].

With the relatively limited data currently available on perinatal vitamin B12 status, we used secondary data from two recent randomized control studies [13,14] to assess the vitamin B12 status of mothers, their breast milk, and their infants in two regions with differing perinatal supplementation and dietary patterns: Canada and Cambodia. We also explored associations between vitamin B12 concentrations in maternal blood, breast milk, and infant blood samples.

\section{Materials and Methods}

\subsection{Study Populations and Biological Sample Collection}

This was a secondary analysis of two studies conducted with exclusively breastfeeding mother-infant dyads in Vancouver, British Columbia, Canada (Canada sample), and Prey Veng province, Cambodia (Cambodian sample). In both samples, a short demographic questionnaire was conducted among participants to gather information such as age, antenatal care, and socioeconomic status; the questionnaire was self-administered in Canada, and interviewer-administered in Cambodia.

\subsubsection{Canadian Sample}

Participants in the Canadian sample were enrolled as part of a randomized control trial from June 2010 through March 2013, investigating the efficacy of three doses of maternal perinatal vitamin $\mathrm{D}$ to improve infant 25-hydroxyvitamin D concentrations at 8 weeks postpartum; results are published elsewhere [13]. Briefly, healthy pregnant women 18-42 years with a low-risk singleton pregnancy in Vancouver, British Columbia were recruited using convenience sampling. Women in this study were randomized to consume an identical daily perinatal multivitamin supplement containing $12 \mu \mathrm{g}$ vitamin B12 starting at 13 to 22 weeks gestation through to 8 weeks post-partum, and a second daily supplement that contained 10, 25, or $50 \mu \mathrm{g}$ vitamin D3. This study was approved by the British Columbia Children's and Women's Clinical Research Ethics Board (H13-01971). 
At 8 weeks postpartum, non-fasting venous blood samples were collected from mothers and infants into trace element-free evacuated tubes (Vacutainer, Becton Dickinson, Mississauga, ON, Canada). The samples were allowed to clot at room temperature for $30 \mathrm{~min}$, centrifuged $(2000 \times g$ for $10 \mathrm{~min}$ at $4{ }^{\circ} \mathrm{C}$ ), and then the serum was removed, aliquoted, and stored at $-80^{\circ} \mathrm{C}$ until analysis. Breast milk samples were collected either at the clinic during the phlebotomy visit or by women themselves the morning of the phlebotomy visit. Regardless of collection site, milk from one full breast expression was collected using an electric breast pump (the woman's own, or Swing breast pump, Medela), more than two hours after the previous feeding. If women collected milk themselves at home, the sample was stored in their refrigerator until the phlebotomy visit $(<10 \mathrm{~h})$. At the clinic, the volume of the milk sample was recorded, and then mixed thoroughly, aliquoted, and stored at $-80{ }^{\circ} \mathrm{C}$ until analysis.

\subsubsection{Cambodian Sample}

Participants in the Cambodian sample were recruited as part of a randomized control trial designed to test the efficacy of maternal ad libitum consumption of thiamin-fortified or control (non-thiamin fortified) fish sauce to improve maternal and infant erythrocyte thiamin diphosphate concentrations and breast milk thiamin concentrations [14]. Healthy women 18-45 years with a low-risk singleton pregnancy residing in Prey Veng province, Cambodia, were recruited between 3-8 months gestation (self-report), and consumed fish sauce for 6 months as part of this study. All women received 90 iron-folic acid supplements during pregnancy; no participants consumed vitamin B12-containing supplements. The National Ethics Committee for Health Research in Cambodia approved this study (0245 NECHR; 386 NECHR).

Between 3-27 weeks postpartum (in April 2015), trained phlebotomists collected non-fasting venous blood samples from mothers and their infants into EDTA-coated tubes (Vacutainer, Becton Dickinson). The samples were placed immediately on ice, and were transported to the National Institute of Public Health (NIPH) laboratory in Phnom Penh within $5 \mathrm{~h}$ of collection. Samples were centrifuged (3000 rpm for $15 \mathrm{~min}$ at $4{ }^{\circ} \mathrm{C}$ ), and plasma removed, aliquoted, and stored at $-80{ }^{\circ} \mathrm{C}$. Breast milk samples were collected during the phlebotomy visit using a procedure identical to that in the Canadian study. Breast milk samples were placed on ice and transported to the NIPH laboratory with the blood samples. Total milk volume was recorded, then each sample was mixed thoroughly, aliquoted into amber cryovials, and stored at $-80{ }^{\circ} \mathrm{C}$. Both blood and breast milk samples were shipped on dry ice to The University of British Columbia in Vancouver, Canada, for storage at $-80^{\circ} \mathrm{C}$ until being shipped to other laboratories for analysis.

\subsection{Biological Sample Analysis}

Serum/plasma samples were shipped on dry ice to the University of Otago in Dunedin, New Zealand for analysis in the Houghton Lab. Total vitamin B12 concentration was measured using an electrochemiluminescence immunoassay (Roche Diagnostics) on an Elecsys 2010 (Roche, New Zealand). Control samples fell within the recommended manufacturer detection range of 22-1495 pmol/L; inter-assay coefficient of variance of a pooled serum sample with a known vitamin B12 concentration within the detection range was $8.3 \%(n=13)$. Total vitamin B12 concentration was measured in serum samples from 124 Canadian mothers and 102 infants; and in plasma samples from 69 Cambodian mothers and 50 infants.

Human milk vitamin B12 concentrations were measured using chemiluminescence on an IMMULITE automated analyzer in the Allen Lab, US Department of Agriculture/ARS Western Human Nutrition Research Centre, Davis; methods reported in detail elsewhere [15]. Milk samples were boiled in dithiothreitol and potassium cyanide to release protein-bound vitamin B12 before analysis. Breast milk vitamin B12 concentration was measured in 109 Canadian and 59 Cambodian samples. 


\subsection{Statistical Analysis}

Only those mothers who self-reported exclusive breastfeeding were included in the analysis. Demographic characteristics are expressed as mean $(95 \% \mathrm{CI})$ and $n(\%)$ for continuous and categorical variables, respectively, unless otherwise noted. Concentrations of vitamin B12 were not normally distributed in either sample for any of the biological specimens $(p<0.05$ for Shapiro-Wilk test of normality), and thus were transformed using the natural log for analyses and back-transformed and expressed as geometric mean $(95 \% \mathrm{CI})$. Independent sample $t$-tests were used to assess differences in maternal age, parity, and maternal, milk, and infant total vitamin B12 concentrations, and chi-square tests were used to assess differences in educational attainment and household income between the Canadian and Cambodian samples. Pearson's bivariate correlations (Canada) and partial correlations (Cambodia, controlled for infant age) were used to determine correlations between maternal and infant total vitamin B12 concentrations, and between milk vitamin B12 concentrations and both maternal and infant total vitamin B12 concentrations.

All infants in the Canadian sample were evaluated at 2 months \pm 1 week of age; infants in the Cambodian sample ranged from 3 to 27 weeks of age, with a mean (SD) age at sample collection of 15 (7) weeks. Therefore, linear regression models were run in the Cambodian sample to assess the effect of infant age on maternal, infant, and milk vitamin B12 concentrations.

Vitamin B12 deficiency is defined here as serum/plasma total vitamin B12 concentration $<148 \mathrm{pmol} / \mathrm{L}$, and marginal deficiency between 148-221 pmol/L [1]. Since there are no cut-offs specifically designed for use in infants, the same cut-offs were employed for both mothers and infants. Chi-square tests were used to determine if there was a difference in the prevalence of serum/plasma vitamin B12 deficiency between groups.

All analyses were performed on SPSS for Macintosh version 23.0 (IBM Corp, Armonk, NY, USA), with a significance level of $p<0.05$.

\section{Results}

All Cambodian mothers were Khmer; in Canada, the majority of mothers $(n=97 ; 79 \%)$ were of European descent ( $7 \%$ were Chinese, and $14 \%$ were of 12 other ethnicities). Canadian mothers were older than the Cambodian mothers: mean $(95 \% \mathrm{CI})$ age was $37(36,38)$ and $26(25,27)$ years, respectively $(p<0.001)$. The Canadian mothers also had greater educational attainment and annual household income than the Cambodian participants $(p<0.001)$. Approximately half of mothers- $53 \%$ in Canada and $54 \%$ in Cambodia-were pregnant for the first time $(p=0.66)$.

Biochemical vitamin B12 status is presented in Table 1. Maternal and infant serum/plasma and breast milk total vitamin B12 concentrations were all significantly higher among the Canadian as compared to the Cambodian sample $(p<0.05)$, although the distributions of mothers' and infants' B12 status did not differ between groups. Histograms showcasing the maternal, milk, and infant total vitamin B12 concentrations of the Canadian and Cambodian samples are shown in Figure 1.

Correlations between maternal, infant, and breast milk total vitamin B12 concentrations are shown in Table 2. In the Canadian sample, all three variables were significantly correlated with one another $(p<0.05)$. In the Cambodian sample, however, only maternal and infant total vitamin B12 concentrations were correlated $(p<0.001)$; breast milk total vitamin B12 concentrations were not correlated with either maternal $(p=0.43)$ or infant $(p=0.64)$ total vitamin B12 concentrations.

Unlike in Canada, where all samples were collected at 2 months post-partum, data and sample collection in Cambodia took place when infants were aged between 3 and 27 weeks, or mean (SD) of 15 (7) weeks postpartum. We used linear regression models to examine the association between infant age and plasma and milk vitamin B12 concentrations among the Cambodian sample, and found that none of these vitamin B12 measurements were significantly correlated with infant age (Table 3). While infant age was borderline inversely associated with milk vitamin B12 concentrations $(p=0.05)$, age would have accounted for $<5 \%$ of the variance in milk B12 concentrations. Table 4 displays the prevalence of vitamin B12 inadequacy among the Cambodian sample by infant age; 8 weeks was used 
as a cut-off to align with the Canadian sample. Again, infant age appeared to influence only milk vitamin B12 concentrations, with older infants (>8 weeks) tending to have lower mean concentrations.

Table 1. Vitamin B12 status among mothers and their infants in Vancouver, Canada, and Prey Veng province, Cambodia ${ }^{1,2}$.

\begin{tabular}{cccccc}
\hline Vitamin B12 Marker & $\boldsymbol{n}$ & Vancouver, Canada & $\boldsymbol{n}$ & Prey Veng, Cambodia & $\boldsymbol{p}$ Value \\
\hline Maternal vitamin B12 & 124 & $698(648,747)$ & 69 & $620(552,687)$ & 0.009 \\
\hline Deficient $(<148)$ & & - & $1(\sim 1 \%)$ & \\
Marginal $(\geq 148$ to 221$)$ & & $1(1 \%)$ & $1(\sim 1 \%)$ & 0.37 \\
Sufficient $(\geq 221)$ & & $123(99 \%)$ & & $67(97 \%)$ & \\
\hline Infant vitamin B12 & 102 & $506(459,552)$ & 50 & $357(312,402)$ & $<0.001$ \\
\hline Deficient $(<148)$ & & $2(2 \%)$ & $4(8 \%)$ & \\
Marginal $(\geq 148$ to 221$)$ & & $4(4 \%)$ & $42(84 \%)$ & 0.10 \\
Sufficient $(\geq 221)$ & & $96(94 \%)$ & & $317(256,378)$ & $<0.001$ \\
\hline Breast milk vitamin B12 ${ }^{3}$ & 109 & $452(400,504)$ & 59 & & \\
\hline
\end{tabular}

${ }^{1}$ Serum (Canada) and plasma (Cambodia) total vitamin B12 (pmol/L), and breast milk vitamin B12 (pmol/L) expressed as geometric mean (95\% CI); vitamin B12 status expressed as $n(\%) ;{ }^{2}$ Independent samples $t$-tests and chi-square tests were employed to assess differences between samples from Canadians and Cambodians of vitamin B12 concentrations and adequacy status categories, respectively; ${ }^{3}$ Breast milk samples collected at 2 months postpartum among Canadian women, and between 3-27 weeks (mean (SD) was 15 (7) weeks) postpartum in the Cambodian sample.

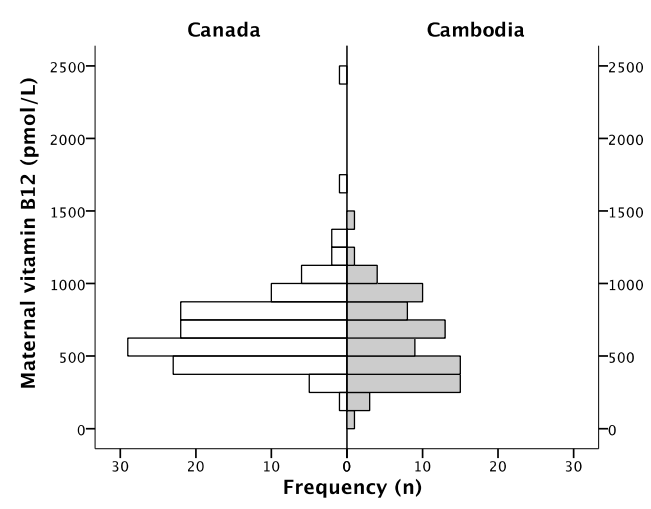

(a)

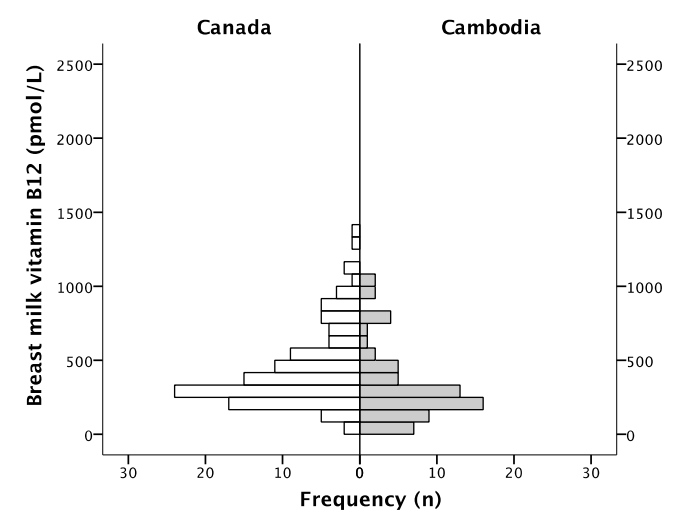

(b)

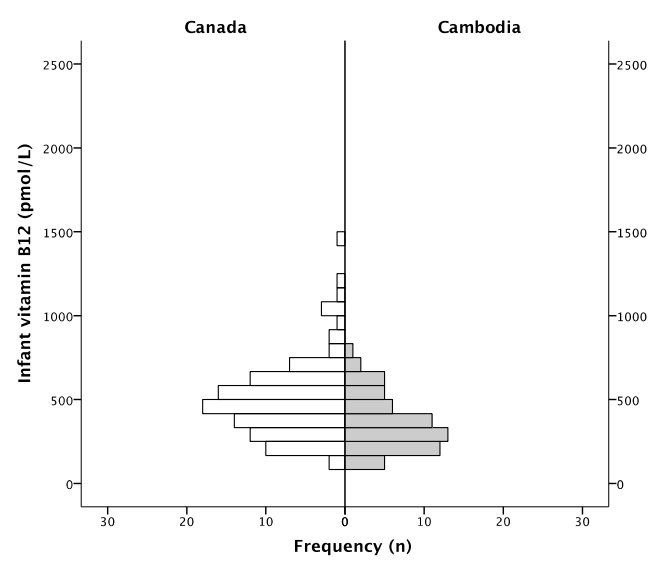

(c)

Figure 1. Histograms of (a) maternal total vitamin B12 concentrations (pmol/L), (b) human milk total vitamin B12 concentrations ( $\mathrm{pmol} / \mathrm{L}$ ), and (c) infant total vitamin B12 concentrations (pmol/L) among the Canadian (white bars) and Cambodian (shaded bars) samples. 
Table 2. Correlations between maternal, infant, and milk total vitamin B12 concentrations among samples of mother-infant dyads in Canada and Cambodia.

\begin{tabular}{cccc}
\hline Vitamin B12 marker & $n$ & Correlation $^{\mathbf{1}}$ & $\boldsymbol{p}$ Value \\
\hline $\begin{array}{c}\text { Maternal vitamin B12 and } \\
\text { breast milk vitamin B12 }\end{array}$ & & & \\
$\quad$ Vancouver, Canada & 109 & 0.498 & $<0.001$ \\
Prey Veng, Cambodia & 59 & 0.105 & 0.43 \\
\hline Maternal vitamin B12 and & & & \\
$\quad$ infant vitamin B12 & & & \\
$\quad$ Vancouver, Canada & 102 & 0.208 & 0.04 \\
Prey Veng, Cambodia & 49 & 0.562 & $<0.001$ \\
\hline Breast milk vitamin B12 and & & & \\
infant vitamin B12 & & & \\
Vancouver, Canada & 88 & 0.370 & $<0.001$ \\
Prey Veng, Cambodia & 45 & 0.073 & 0.64 \\
\hline
\end{tabular}

${ }^{1}$ Pearson's bivariate correlations were used for the Canadian sample; partial correlations controlling for infant age were used in the Cambodian sample.

Table 3. Linear regression models of the association between infant age and plasma and milk vitamin B12 concentrations among the Cambodian sample.

\begin{tabular}{cccccc}
\hline Vitamin B12 Marker & Adjusted $\boldsymbol{R}^{\mathbf{2}}$ & \multicolumn{2}{c}{ Unstandardized } & \multirow{2}{*}{ Standardized $\beta$} & \multirow{2}{*}{$\boldsymbol{p}$ Value } \\
\cline { 3 - 4 } & & $\boldsymbol{\beta}$ & $\mathbf{9 5 \%} \mathbf{C I}$ & & \\
\hline $\begin{array}{c}\text { Maternal plasma } \\
\text { vitamin B12 }\end{array}$ & 0.038 & $-0.015-0.031,0.001$ & -0.229 & 0.06 \\
$\begin{array}{c}\text { Milk vitamin B12 } \\
\text { Infant plasma } \\
\text { vitamin B12 }\end{array}$ & 0.049 & $-0.028-0.056,0.000$ & -0.256 & 0.05 \\
\hline
\end{tabular}

Table 4. Total vitamin B12 concentrations and prevalence of vitamin B12 sufficiency by infant age (older or younger than 8 weeks) among the Cambodian sample ${ }^{1}$.

\begin{tabular}{|c|c|c|c|c|c|}
\hline Vitamin B12 Marker & $n$ & Infant $\leq 8$ weeks & $n$ & Infant $>8$ weeks & $p$ Value \\
\hline Maternal plasma vitamin B12 & & $634(464,803)$ & & $615(541,688)$ & 0.85 \\
\hline Deficient $(<148)$ & & - & & - & \\
\hline Marginal ( $\geq 148$ to 221$)$ & 18 & - & 51 & $1(2 \%)$ & 0.73 \\
\hline Sufficient $(\geq 221)$ & & $18(100 \%)$ & & $50(98 \%)$ & \\
\hline Infant plasma vitamin B12 & 12 & $343(253,433)$ & 38 & $361(307,415)$ & 0.93 \\
\hline Deficient $(<148)$ & & - & & $4(10 \%)$ & \\
\hline Marginal $(\geq 148$ to 221$)$ & & $1(7 \%)$ & & $3(8 \%)$ & 0.50 \\
\hline Sufficient $(\geq 221)$ & & $11(93 \%)$ & & $31(82 \%)$ & \\
\hline Breast milk vitamin B12 ${ }^{2}$ & 13 & $427(266,588)$ & 46 & $286(222,351)$ & 0.051 \\
\hline
\end{tabular}

\section{Discussion}

Milk vitamin B12 concentrations reported in the literature vary considerably. Greibe and colleagues reported median concentrations of vitamin B12 in the hindmilk of 25 well-nourished Danish mothers of 760, 290, and $440 \mathrm{pmol} / \mathrm{L}$ at 2 weeks, 4 months, and 9 months postpartum, respectively [11], indicating variability with lactation stage. In this study, we found higher total vitamin 
B12 concentrations in the milk of Cambodian mothers $\leq 8$ weeks postpartum $(n=13)$ compared with $>8$ weeks postpartum $(n=46)$ ( 427 versus $286 \mathrm{pmol} / \mathrm{L}$, respectively), however this was not statistically significant ( $p=0.051$; Table 4$)$. Regardless, these results are difficult to compare due to the low sample sizes, differing time-points for milk collection, and because we collected a full milk expression, while only hindmilk was collected in the Danish study.

As noted earlier, a milk vitamin B12 deficiency cut-off of $<362$ pmol/L exists [9,10], but is not commonly employed due to potential overestimation of inadequacy. Nearly all mothers in both Canada and Cambodia ( $\geq 97 \%$ ) had adequate vitamin B12 status ( $>221 \mathrm{pmol} / \mathrm{L})$, as did their infants-94\% and $84 \%$, respectively. However, the vitamin B12 status of breast milk was below the cut-off of $362 \mathrm{pmol} / \mathrm{L}[9,10]$ among 50\% and 75\% of Canadian and Cambodian mothers, respectively (data not shown). The high prevalence of adequate vitamin B12 status among infants despite less than adequate breast milk vitamin B12 levels highlights the necessity for more research to better define deficient vitamin B12 breast milk levels given current laboratory methods.

Consistent with previous studies, we found significant correlations between maternal and infant serum/plasma total vitamin B12 concentrations in both the Canadian and Cambodian samples. For example, Greibe et al. identified significant correlations $(p<0.05)$ between maternal and infant vitamin B12 concentrations at three different time-points among Danish infants aged 2 weeks $(r=0.52), 4$ months $(r=0.47)$, and 9 months $(r=0.29)$ [11]. Similarly, significant correlations between maternal and infant plasma vitamin B12 were reported among Malawian dyads $(n=521)$ in the Breastfeeding, Antiretroviral, and Nutrition (BAN) study-r $=0.42$ and 0.32 at 2 or 6 weeks and 24 weeks, respectively [16].

In our study, we found that milk vitamin B12 concentration was not significantly correlated with infant total vitamin B12 in the Cambodian sample; however, at 2 months post-partum, milk was significantly but weakly correlated with infant serum vitamin B12 concentrations in the Canadian sample $(r=0.108, p<0.001$; see Table 2). The vitamin B12 content of milk is impacted by maternal dietary vitamin B12 intake and depletion [5]; however, the association between milk vitamin B12 and infant vitamin B12 status is not consistent. Greibe et al. found that milk was only significantly correlated with infant plasma vitamin B12 concentrations at 4 months postpartum $(r=0.58 ; p=0.005)$ but not 2 weeks or 9 months [11]. In a Guatemalan study of 113 dyads at 3 months postpartum, the authors reported that milk vitamin B12 concentrations were inversely associated with infant vitamin B12 status $(r=-0.22 ; p<0.05)$ as assessed by urinary methylmalonic acid [10]. The BAN study in Malawi reported significant correlations between maternal plasma, breast milk, and infant plasma vitamin B12 concentrations at both 2 or 6 and 24 weeks, but speculated that maternal vitamin B12 status in pregnancy is likely a more important predictor of infant status than milk concentrations [16]. It may be that maternal vitamin B12 status during pregnancy is a better indicator of infant vitamin B12 adequacy in the first year of life than milk vitamin B12 concentrations, given that infants of well-nourished mothers are born with $\sim 25 \mu \mathrm{g}$ of vitamin B12 stores [17], which should protect the infant from inadequacy to 12 months of age regardless of milk vitamin B12 content.

Maternal and infant serum/plasma and breast milk total vitamin B12 concentrations were significantly higher among the Canadian sample compared to the Cambodian sample $(p<0.05)$. These differences were not unexpected, because the Canadian mothers were consuming a daily vitamin B12-containing perinatal supplement providing $>400 \%$ of the recommended dietary allowance for lactation [18]. Although all Cambodian mothers were consuming fish sauce as part of the original randomized control trial [14], this is not a significant source of dietary vitamin B12; American fish sauce provides only $\sim 0.05 \mu \mathrm{g}$ vitamin B12 per $10 \mathrm{~mL}$ fish sauce [19] as per international CODEX standards [20]. The lack of vitamin B12 deficiency in Cambodia is consistent with the most recent Cambodian Demographic and Health Survey (2014), which reported only $1 \%$ of mothers $(n=731)$ were vitamin B12 deficient ( $<150 \mathrm{pmol} / \mathrm{L})$ [21].

This study has several strengths, namely the collection of three biological samples from mother-infant dyads at the same time-point (mother and infant blood, and breast milk). In addition, 
a full breast expression was collected, and samples were collected from the majority of women first thing in the morning, which helps to limit intra-participant diurnal variation, although this is not a major concern with vitamin B12 [22]. We also employed current laboratory methods to assess milk vitamin B12 [15]; older methods may have incorrectly measured vitamin B12 analogues. However, as a secondary analysis, this study has several shortfalls. It would have been ideal to measure at least one direct and one functional indicator for vitamin B12 status in addition to total vitamin B12 concentration. However, due to a lack of blood samples and funding, we were unable to measure methylmalonic acid or total homocysteine. We selected total vitamin B12 concentration as our indicator because it is the most commonly employed and inexpensive direct biomarker [23]. The lack of longitudinal data is a limitation, especially given that vitamin B12 levels appear to fluctuate throughout lactation. In addition, breast milk values from Cambodia cannot be directly compared against those from Canada because they were not collected at the same time; while early literature indicates that breast milk B12 concentrations remain relatively constant after falling from their highest levels in colostrum [12], a more recent study reported fluctuation throughout lactation [11]. Our analyses included only exclusively breastfeeding dyads, and therefore it is less relevant for comparison with children consuming breast milk substitutes or those who have started consuming complementary foods. Dietary data were not collected, limiting our ability to generalize these findings to populations with known high- or low-vitamin B12 intakes. Anthropometric measures were not assessed at the time of blood and milk collection in either group. In addition, these samples were not representative, limiting generalizability.

\section{Conclusions}

The vast majority of both Canadian and Cambodian mothers $(\geq 97 \%)$ and infants $(\geq 84 \%)$ had sufficient vitamin B12 status ( $>221$ pmol/L), however maternal, infant, and milk total vitamin B12 concentrations were significantly higher among the Canadian sample compared to the Cambodians. In the Canadian sample, maternal, milk, and infant vitamin B12 concentrations all significantly correlated, while only maternal and infant plasma vitamin B12 concentrations were correlated in the Cambodian sample. The wider age range among Cambodian infants ( 3 to 27 weeks) allowed for the exploration of associations in infant age revealing a borderline significant inverse relationship with infant age and infant B12 status. The vast majority of both mothers $(\geq 97 \%)$ and infants $(\geq 84 \%)$ in both groups had sufficient vitamin B12 status (>221 pmol/L).

Acknowledgments: Funding: A Grand Challenges Canada Stars in Global Health Round VI Phase I grant (S6 0490-01-10) funded the Cambodian study, and the Canadian study was funded by the Canadian Institutes of Health Research. KCW gratefully acknowledges funding support through the International Development Research Centre Doctoral Research Award, and K.C.W. and K.M.M. were supported by Canadian Institutes of Health Research Frederick Banting and Charles Best Canada Graduate Scholarships. We thank the following individuals from Helen Keller International, Cambodia: Sim Chhoeun and Phon Phearom for help with recruitment, field coordination, and data collection, Rem Ngik for data collection and management, and Zaman Talukder, Ame Stormer and Keith Porter for invaluable input into aspects of field implementation. We thank Chanthan Am and the field and laboratory teams at the National Institute of Public Health (Phnom Penh, Cambodia) for blood collection and processing.

Author Contributions: Study conception and design: T.J.G., S.I.B., K.C.W., M.B., P.S., H.K., Y.L.; study coordination and data collection: K.M.M., N.N.C., H.C., M.B., P.S., K.C.W.; laboratory method development and biological sample analysis: R.A.S., S.S.-F., D.H., L.A.H., L.H.A.; data analysis: P.C., C.D.K., K.C.W., T.J.G.; drafted manuscript: P.C., K.C.W., T.J.G.; contributed to the review and revision of the manuscript, and read and approved the final manuscript: all authors.

Conflicts of Interest: The authors declare no conflict of interest.

\section{References}

1. Allen, L.H. How common is vitamin B-12 deficiency? Am. J. Clin. Nutr. 2009, 89, 693S-696S. [CrossRef] [PubMed]

2. Dror, D.K.; Allen, L.H. Effect of vitamin B12 deficiency on neurodevelopment in infants: Current knowledge and possible mechanisms. Nutr. Rev. 2008, 66, 250-255. [CrossRef] [PubMed] 
3. Williams, A.M.; Chantry, C.J.; Young, S.L.; Achando, B.S.; Allen, L.H.; Arnold, B.F.; Colford, J.M.; Dentz, H.N.; Hampel, D.; Kiprotich, M.C.; et al. Vitamin B-12 concentrations in breast milk are low and are not associated with reported household hunger, recent animal-source food, or vitamin B-12 intake in women in rural Kenya. J. Nutr. 2016, 146, 1125-1131. [CrossRef] [PubMed]

4. Deegan, K.L.; Jones, K.M.; Zuleta, C.; Ramirez-Zea, M.; Lildballe, D.L.; Nexo, E.; Allen, L.H. Breast Milk Vitamin B-12 Concentrations in Guatemalan Women Are Correlated with Maternal but Not Infant Vitamin B-12 Status at 12 Months Postpartum. J. Nutr. 2012, 142, 112-116. [CrossRef] [PubMed]

5. Allen, L.H. B vitamins in breast milk: Relative importance of maternal status and intake, and effects on infant status and function. Adv. Nutr. 2012, 3, 362-369. [CrossRef] [PubMed]

6. Kramer, M.S.; Kakuma, R. The Optimal Duration of Exclusive Breastfeeding: A Systematic Review; World Health Organization: Geneva, Switzerland, 2002.

7. Molloy, A.M.; Kirke, P.N.; Brody, L.C.; Scott, J.M.; Mills, J.L. Effects of folate and vitamin B 12 deficiencies during pregnancy on fetal, infant, and child development. Food Nutr. Bull. 2008, 29, S101-S111. [CrossRef] [PubMed]

8. Carmel, R. Cobalamin (vitamin B12). In Modern Nutrition in Health and Disease; Ross, A.C., Caballero, B., Cousins, R.J., Tucket, K.L., Ziegler, T.R., Eds.; Lippincott Williams \& Wilkins: Baltimore, MD, USA, 2014; pp. 369-389.

9. Specker, B.L.; Black, A.; Allen, L.H.; Morrow, F. Vitamin B-12: Low milk concentrations are related to low serum concentrations in vegetarian women and to methylmalonic aciduria in their infants. Am. J. Clin. Nutr. 1990, 52, 1073-1076. [PubMed]

10. Casterline, J.E.; Allen, L.H.; Ruel, M.T. Vitamin B-12 deficiency is very prevalent in lactating Guatemalan women and their infants at three months postpartum. J. Nutr. 1997, 127, 1966-1972. [PubMed]

11. Greibe, E.; Lildballe, D.L.; Streym, S.; Vestergaard, P.; Rejnmark, L.; Mosekilde, L.; Nexo, E. Cobalamin and haptocorrin in human milk and cobalamin-related variables in mother and child: A 9-mo longitudinal study. Am. J. Clin. Nutr. 2013, 98, 389-395. [CrossRef] [PubMed]

12. Trugo, N.M.; Donangelo, C.M.; Koury, J.C.; Silva, M.I.; Freitas, L.A. Concentration and distribution pattern of selected micronutrients in preterm and term milk from urban Brazilian mothers during early lactation. Eur. J. Clin. Nutr. 1988, 42, 497-507. [PubMed]

13. March, K.M.; Chen, N.N.; Karakochuk, C.D.; Shand, A.W.; Innis, S.M.; von Dadelszen, P.; Barr, S.I.; Lyon, M.R.; Whiting, S.J.; Weiler, H.A.; et al. Maternal vitamin D3 supplementation at $50 \mathrm{ug} / \mathrm{d}$ protects against low serum 25-hydroxyvitamin D in infants at $8 \mathrm{wk}$ of age: A randomized controlled trial of 3 doses of vitamin D beginning in gestation and continued in lactation. Am. J. Clin. Nutr. 2015, 102, 402-410. [CrossRef] [PubMed]

14. Whitfield, K.C.; Karakochuk, C.D.; Kroeun, H.; Hampel, D.; Sokhoing, L.; Chan, B.B.; Borath, M.; Sophonneary, P.; McLean, J.; Talukder, A.; et al. Perinatal consumption of thiamine-fortified fish sauce in rural Cambodia: A randomized clinical efficacy trial. JAMA Pediatr. 2016, 170, e162065. [CrossRef] [PubMed]

15. Hampel, D.; Shahab-Ferdows, S.; Domek, J.M.; Siddiqua, T.; Raqib, R.; Allen, L.H. Competitive chemiluminescent enzyme immunoassay for vitamin B12 analysis in human milk. Food Chem. 2014, 153, 60-65. [CrossRef] [PubMed]

16. Shahab-Ferdows, S.; Allen, L.H.; Hampel, D.; Bentley, M.E.; Adair, L.S.; Flax, V.L.; Chasela, C.S.; Kacheche, Z.; Tegha, G.; Kourtis, A.P.; et al. Maternal and breastmilk vitamin B12 correlated with infant status but was not influenced by maternal supplementation among HIV-infected Malawian women in the Breastfeeding, Antiretrovirals and Nutrition Study (BAN). FASEB J. 2013, 27, 107.2.

17. Allen, L.H. Vitamin B12 metabolism and status during pregnancy, lactation and infancy. In Nutrient Regulation during Pregnancy, Lactation, and Infant Growth; Allen, L.H., King, J., Lonnerdal, B., Eds.; Premium Press: New York, NY, USA, 1994; pp. 173-186.

18. Institute of Medicine Vitamin B12. In Dietary Reference Intakes for Thiamin, Riboflavin, Niacin, Vitamin B6, Folate, Vitamin B12, Pantothenic Acid, Biotin, and Choline; National Academy Press: Washington, DC, USA, 1998; pp. 306-356.

19. United States Department of Agriculture (USDA). Ready-to-Serve Fish Sauce (06179). Available online: https: / / ndb.nal.usda.gov/ndb/foods/show /1311?fgcd=\&manu=\&lfacet=\&format=\&count=\&max=50\& offset $=\&$ sort $=$ default\&order $=$ asc\&qlookup $=$ fish + sauce $\& d s=($ accessed on 3 October 2016). 
20. Codex Alimentarius International Food Standards. Standard for Fish Sauce (CODEX STAN 302-2011); Amendment 2012; Codex Alimentarius International Food Standards: Geneva, Switzerland, 2011.

21. National Institute of Statistics; Directorate General for Health; ICF International. Cambodia Demographic and Health Survey 2014; National Institute of Statistics: Phnom Penh, Cambodia; Rockville, MD, USA, 2015.

22. Hampel, D.; Shahab-Ferdows, S.; Islam, M.M.; Peerson, J.M.; Allen, L.H. Vitamin concentrations in human milk vary with time within feed, circadian rhythym, and single-dose supplementation. J. Nutr. 2017. [CrossRef] [PubMed]

23. Fedosov, S.N.; Brito, A.; Miller, J.W.; Green, R.; Allen, L.H. Combined indicator of vitamin B12 status: modification for missing biomarkers and folate status and recommendations for revised cut-points. Clin. Chem. Lab. Med. 2015, 53, 1215-1225. [CrossRef] [PubMed]

(C) 2017 by the authors. Licensee MDPI, Basel, Switzerland. This article is an open access article distributed under the terms and conditions of the Creative Commons Attribution (CC BY) license (http:/ / creativecommons.org/licenses/by/4.0/). 